\title{
Updated consensus statement on tumour necrosis factor blocking agents for the treatment of rheumatoid arthritis (May 2000)
}

As last year, a consensus group to consider the use of tumour necrosis factor (TNF) blocking agents was formed by an organising committee constituted of rheumatologists from the Universities of Erlangen, Leiden and Vienna in Europe in cooperation with universities in the United States. Pharmaceutical support was obtained from a number of companies, but these institutions had no part in the decisions regarding this specific programme nor with regard to the participants or attendees at this conference.

The approximately 100 rheumatologists and bioscientists from 25 countries who attended the consensus conference were chosen from a worldwide group of people felt to have experience or interest in the use of TNF blocking treatment for rheumatoid arthritis (RA). Unfortunately, the number of attendees and participants were limited so that not everyone who might have been appropriate could be invited.

During the past year increasing amounts of data on the use of TNF blocking agents in RA have been published. Consequently it was felt appropriate to update the provisional consensus statement published last year (Ann Rheum Dis 1999;58 (suppl 1):I129-30).

During this revision it became clear that there are still large areas where knowledge is lacking. In an effort to clarify, for the readers, evidence on which the consensus statement is based, statements with supporting data have been referenced.

When a statement is not referenced it will have arisen from the consensus process: small group discussions, large group discussions and repeated drafts of the consensus statement to allow input from all participants. We feel that this statement represents an updated, although still provisional, consensus view of the use of TNF blockade in RA. However, it will require continued updating, and upgrading, as more evidence is gathered, presented in scientific forums and discussed.

While all TNF blocking agents are not alike (they differ in composition, precise mechanisms of action, pharmacokinetics, biopharmaceutical properties, etc) this consensus statement will emphasise areas of commonality and areas where knowledge is missing. ${ }^{12}$ If, and when, data differentiating compounds require discussion, they will be considered in the future, based on the persuasiveness of the data and the degree of difference demonstrated.

To help the reader understand the differences between last year's and this year's consensus statements, significant changes will be highlighted. Some statements have been altered to aid the flow or logic of the consensus statement, although their content and sense remains unchanged. Those statements will not be highlighted. Some statements have been dropped out. Those will not be noted in the present statement. Readers are urged to compare the two statements if they are interested in the latter.

Finally, to help point the way forward, the participants have presented areas where research is needed to help rheumatologists understand and use TNF blocking agents more rationally and effectively.

\section{Indications}

TNF blocking agents should normally be used for the treatment of active RA or juvenile chronic arthritis (JCA) after a full trial of an effective DMARD - of which methotrexate is a commonly used example-has been shown to be inadequate. ${ }^{3-5}$ Individual patients differ in the aggressiveness of their disease and its concomitant structural damage, the effect of their disease on their quality of life and the symptoms and signs engendered by their disease. All these factors must be examined when considering TNF treatment for that patient, as must the toxicity of previous DMARD use. TNF blocking agents can be added to pre-existing treatment or, when appropriate, may replace previous DMARDs. ${ }^{45}$ Initial controlled studies have shown that these agents are efficacious in rheumatoid arthritis (RA) before the use of conventional DMARDS. ${ }^{6}$ Pharmacoeconomic data and long term safety data may change the circumstances when TNF blocking agents will be started, added to, or replace other DMARDs (studies in progress).

\section{Reasons for discontinuation}

TNF blocking agents, when given in adequate doses and sufficiently frequent dosing regimens, should lead to significant, documentable improvement in symptoms, signs and/or laboratory parameters within 4-16 weeks. ${ }^{3-7}$ Quantifiable disease activity measures (such as tender and swollen joint counts, modified Health Assessment Questionnaires (HAQ), Visual Analogue Scales (VAS) for global response by patient or physician, VAS for pain, laboratory data or combined parameters of response) should be followed and a substantial response to treatment must be demonstrated in that time frame..$^{1-7}$ If such improvement has not occurred within that time frame, alternative treatments or regimens should be considered. Although there are data supporting the slowing of radiological progression of TNF blocking agents, the implication as well as clinical significance of this finding remains to be determined and requires further studies. ${ }^{6-8}$ Until such additional studies are completed radiological changes alone are insufficient by themselves to drive clinical decision making.

\footnotetext{
Warnings

TNF blocking agents should not be started or should be discontinued when serious infections occur, such as septic arthritis, infected prosthesis, pneumonia, pyelonephritis, acute abscesses, osteomyelitis, sepsis, systemic fungal infections, listeria or mycobacterial infections, etc. ${ }^{10}$ Treatment should only be resumed if such infections have been completely resolved and the risk of recurrence is low.
} 


\section{Precautionary statements}

The effect of TNF blockade is unknown in the following situations:

- Lymphoma, lymphoproliferative and other malignancies.

- Chronic infections, including HIV, hepatitis B or C, mycobacterial disease, etc.

- During pregnancy or lactation.

- When considering primary vaccinations or live attenuated vaccines.

Other areas where knowledge is lacking are highlighted in the consensus groups recommendations for areas most urgently requiring further research.

\section{Additional comments}

Rare cases of lupus-like disease have occurred in patients receiving TNF blocking agents and treatment should be stopped if there is clinical evidence of drug induced lupuslike syndrome. There is thus far no evidence that RA patients who have positive ANA and/or ACL are at increased risk for the development of drug induced lupus.

If TNF blocking agents are proved efficacious and their safety and toxicity profile is demonstrated in patients with polyarticular psoriatic arthritis and ankylosing spondylitis and are approved by appropriate regulatory agencies these treatments could be used to treat those patients. ${ }^{11}$

Among a number of potential areas requiring action and/or further research the consensus group felt the following projects or directions were most important in each of three areas: efficacy, toxicity and general issues:

EFFICACY

- What are the optimum dosing regimens and dosage intervals when using TNF blocking agents; also is intermittent use appropriate?

- Are there predictors of response (and toxicity), or lack thereof, for TNF blocking agents?

- Is there a correlation between radiological effect and long term effectiveness for TNF blocking agents?

- What are the clinically useful outcome measures to monitor anti-TNF administration?

- How does previous or concomitant DMARD treatment affect the efficacy and toxicity of TNF blocking agents?

TOXICITY

- A long term registry to monitor the toxicity and effectiveness of TNF blocking treatment is strongly recommended, requiring a cooperative effort among payors, government, industry and rheumatologists.

- Can patients with evidence of prior mycobacterial infection, or fungal infection, use TNF blocking agents with safety comparable to patients without such a prior history?

- Can TNF blocking agents be used in pregnant or lactating women (and their offspring) as safely as in matched female patients who are not pregnant or lactating?

- Do TNF blocking agents affect the efficacy of primary vaccination or the safety or effects of live attenuated vaccination?

- What is the safety profile of TNF blocking agents during surgery and how does it compare with the safety profile in patients undergoing surgery without concomitant TNF blocker use?

GENERAL USE

There is a need to develop and introduce an instrument that measures and documents quality management in the care of RA patients.

\section{Summary}

TNF blocking agents are promising therapeutic agents for RA. Studies in selected areas of efficacy, toxicity and the general use of TNF blocking agents are needed to help further define the most appropriate use of these agents. The use of these new agents will require physicians experienced in the diagnosis, treatment and assessment of RA. These physicians will need to make long term observations for efficacy and safety. Further considerations when using TNF blocking agents in this disease must include cost issues and recognition that data in subpopulations are still being acquired. It is hoped that this statement, which is based upon the best evidence available at the time of its creation and modified by expert opinion, will facilitate the optimal use of these agents for our patients with RA.

D E FURST F C BREEDVELD G R BURMESTER J J CROFFORD P EMERY M FELDMANN J R KALDEN A F KAVANAUGH E C KEYSTONE L G KLARESKOG P E LIPSKY R N MAINI

A S RUSSELL D L SCOTT

J S SMOLEN

L B A VAN DE PUTTE T L VISHER

M H WEISMAN

1 Markham A, Lamb HM. Infliximab: a review of its use in the management of RA. Drugs 2000;59:1341-59.

2 Garrison L, McDonnell ND. Etanercept: therapeutic use in patients with RA. Ann Rheum Dis 1999;58 (suppl 1):165-9.

3 Maini RN, Breedveld FC, Kalden JR, et al. Therapeutic efficacy of multiple intravenous infusions of anti-tumour necrosis factor (alpha) monoclonal antibody versus placebo in RA patients receiving concomitant methotrexate: a randomized phase III trial. Lancet 1999;354:1932-9.

4 Moreland LW, Baumgartner SW, Schiff MH, et al. Treatment of RA with a recombinant human tumour necrosis factor receptor (p75)-Fc fusion protein. N Engl J Med 1997;337:141-7.

5 Lovell DJ, Giannini EH, Reiff A, et al. Etanercept in children with polyarticular juvenile rheumatoid arthritis. N Engl J Med 2000;342:763-9.

6 Finck B, Martin R, Fleischmann R, et al. A phase III trial of etanercept vs methotrexate in early RA. [Abstract]. Arthritis Rheum 1999;42 (suppl): s117.

7 Ran R, Herborn G, Sander O, et al. Long term treatment with the fully human anti-TNF Antibody D2E7 shows radiographic disease progression in RA. [Abstract]. Arthritis Rheum 1999;42 (suppl):s400

8 Lipsky P, St. Clair W, Furst D, et al. 54 week clinical and radiographic results from the ATTRACT Trial: a phase III study of infliximab in patients with active RA despite methotrexate. [Abstract]. Arthritis Rheum 1999;42 (suppl):s401.

9 Etanercept United States pharmacopoeia drug information. Vol 1. Englewood, CO: Micromedex, 1473-5.

10 Infliximab United States pharmacopoeia drug information. Vol 1. Englewood, CO: Micromedex, 1774-7.

11 Mease P, Goffe B, Venderstoep A. Etanercept in patients with psoriatic arthritis and psoriasis. [Abstract]. Arthritis Rheum 1999;42 (suppl):s377. 